International Journal of Pure and Applied Mathematics

Volume 103 No. 4 2015, 613-624

ISSN: 1311-8080 (printed version); ISSN: 1314-3395 (on-line version)

url: http://www.ijpam.eu

doi: http://dx.doi.org/10.12732/ijpam.v103i4.2

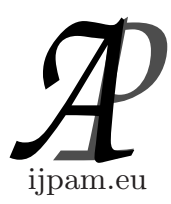

\title{
ON THE CONFORMAL CHANGE OF DOUGLAS SPACE OF SECOND KIND WITH CERTAIN $(\alpha, \beta)$-METRICS
}

\author{
Gauree Shanker ${ }^{1}$, Deepti Choudhary ${ }^{2}$ \\ Department of Mathematics and Statistics \\ Banasthali University \\ Banasthali, Rajasthan, 304022, INDIA
}

\begin{abstract}
The Douglas space of second kind with an $(\alpha, \beta)$-metric was defined by I.Y. Lee [7]. In this paper, we prove that a Douglas space of second kind with an $(\alpha, \beta)$-metric is conformally transformed to a Douglas space of second kind. Further, we find the conditions under which the conformal change of Finsler space with Matsumoto and generalized Kropina metric is of Douglas space of second kind.
\end{abstract}

AMS Subject Classification: 53B40, 53C60

Key Words: conformal change, Douglas space, Douglas space of second kind, Matsumoto metric, generalized Kropina metric

\section{Introduction}

It is well known that a Finsler space with $(\alpha, \beta)$-metric is a Douglas space of second kind if the Douglas tensor $D_{i j k}^{h}$ vanishes identically [3]. S. Bácsó and Matsumoto [2] introduced the notion of a Finsler space with $(\alpha, \beta)$-metric of Douglas type as a generalization of the Berwald space from the viewpoint of geodesic equations. Recently, I. Y. Lee [7] has studied Douglas space of second kind and he has find the conditions for a Finsler space with Matsumoto metric to be a Douglas space of second kind.

Received: March 8, 2015

(c) 2015 Academic Publications, Ltd.

$\S$ Correspondence author url: www.acadpubl.eu 
A Finsler space $F^{n}$ is said to be a Douglas space if $D^{i j}=G^{i}(x, y) y^{j}-$ $G^{j}(x, y) y^{i}$ are homogeneous polynomials in $\left(y^{i}\right)$ of degree three. Then a Finsler space $F^{n}$ is said to be a Douglas space of the second kind if and only if $D_{m}^{i m}=$ $(n+1) G^{i}-G_{m}^{m} y^{i}$ are homogeneous polynomials in $\left(y^{i}\right)$ of degree two. On the other hand, in [11] it has been shown that a Finsler space with $(\alpha, \beta)$-metric is a Douglas space if and only if $B^{i j}=B^{i} y^{j}-B^{j} y^{i}$ are homogeneous polynomials in $\left(y^{i}\right)$ of degree three. Therefore a Finsler space with an $(\alpha, \beta)$-metric is said to be a Douglas space of the second kind if and only if $B_{m}^{i m}=(n+1) B^{i}-B_{m}^{m} y^{i}$ are homogeneous polynomials in $\left(y^{i}\right)$ of degree two, where $B_{m}^{i m}$ is given by [6](Theorem 2.1).

The conformal theory of Finsler space was introduced by M. S. Kneblman in 1929 [5] and it has been investigated in detail by M. Hashiguchi, [4]. Later on Y. D. Lee [8] and B. N. Prasad [14] found conformally invariant tensors in the Finsler space with $(\alpha, \beta)$-metric. In [12], conformal transformation of Douglas space with special $(\alpha, \beta)$-metric have been studied by S. K. Narasimhamurthy.

The purpose of the present paper is to find the conditions for a Douglas space of second kind with $(\alpha, \beta)$-metric to be a Douglas space of second kind under conformal transformation (Theorem 4.2). Further we have proved that Douglas space of second kind with Matsumoto and generalized Kropina metric is a Douglas space of second kind under conformal transformation (Theorem 5.1 and Theorem 5.2).

\section{Preliminaries}

Let $F^{n}=(M, L(\alpha, \beta))$ be an n-dimensional Finsler space, where $M$ is a differential manifold of dimension $\mathrm{n}$ and $L(x, y)$ (where $y^{i}=\dot{x}^{i}$ ) is the fundamental function defined on the slit tangent bundle $T M_{0}=T M \backslash\{0\}$ of manifold $M$. We assume that $L(x, y)$ is positive and the metric tensor $g_{i j}(x)=\frac{1}{2} \dot{\partial}_{j} \dot{\partial}_{i} L^{2}$ is positive definite, where $\dot{\partial}_{i}=\frac{\partial}{\partial y^{i}}$.

The geodesics of an n-dimensional Finsler space $F^{n}=(M, L)$ are given by the system of differential equations [4]

$$
\frac{d^{2} x^{i}}{d t^{2}} y^{j}-\frac{d^{2} x^{j}}{d t^{2}} y^{i}+2\left(G^{i} y^{j}-G^{j} y^{i}\right)=0, y^{i}=\frac{d x^{i}}{d t}
$$

in a parameter $t$. The function $G^{i}(x, y)$ is given by

$$
2 G^{i}(x, y)=g^{i j}\left(y^{r} \dot{\partial}_{j} \partial_{r} F-\partial_{j} F\right)=\gamma_{j k}^{i}(x, y) y^{j} y^{k}
$$


where $\partial_{i}=\frac{\partial}{\partial x^{i}}, F=\frac{L^{2}}{2}, \gamma_{j k}^{i}$ are Christoffel symbols constructed from $g_{i j}(x, y)$ with respect to $x^{i}$ and $g^{i j}(x, y)$ is the inverse of fundamental metric tensor $g_{i j}(x, y)$.

In [2], it has been shown that $F^{n}$ is a Douglas space if and only if the Douglas tensor [3]

$$
D_{i j k}^{h}=G_{i j k}^{h}-\frac{1}{n+1}\left(G_{i j k} y^{h}+G_{i j} \delta_{k}^{h}+G_{j k} \delta_{i}^{h}+G_{k i} \delta_{j}^{h}\right)
$$

vanishes identically, where $G_{i j k}^{h}=\dot{\partial}_{k} G_{i j}^{h}$ is the $h v$-curvature tensor of the Berwald connection $B \Gamma[10]$.

The space $F^{n}$ is said to be a Douglas space [2] if

$$
D^{i j}=G^{i}(x, y) y^{j}-G^{j}(x, y) y^{i}
$$

are homogeneous polynomials in $y^{i}$ of degree three. Differentiating (2.1) with respect to $y^{h}, y^{k}, y^{p}$, and $y^{q}$, we have $D_{h k p q}^{i j}=0$, which are equivalent of $D_{h k p m}^{i m}=(n+1) D_{h k p}^{i}=0$. Thus if a Finsler space $F^{n}$ satisfies the condition $D_{h k p q}^{i j}=0$, which are equivalent to $D_{h k p m}^{i m}=(n+1) D_{h k p}^{i}=0$, we call it a Douglas space. Further differentiating (2.1) by $y^{m}$ and contacting $m$ and $j$ in the obtained equation, we have $D_{m}^{i m}=(n+1) G^{i}-G_{m}^{m} y^{i}$. Thus $F^{n}$ is said to be a Douglas space of the second kind if and only if

$$
D_{m}^{i m}=(n+1) G^{i}-G_{m}^{m} y^{i}
$$

are homogeneous polynomials in $\left(y^{i}\right)$ of degree two. Furthermore differentiating (2.2) with respect to $y^{h}, y^{j}$ and $y^{k}$, we get $D_{h j k m}^{i m}=(n+1) D_{h j k}^{i}=0$. Therefore we have

Definition 2.1. A Finsler space $F^{n}$ is said to be a Douglas space of second kind if $D_{m}^{i m}=(n+1) G^{i}-G_{m}^{m} y^{i}$ is a homogeneous polynomials in $\left(y^{i}\right)$ of degree two.

A Finsler metric $L(x, y)$ is called an $(\alpha, \beta)$-metric, if $L$ is a positively homogeneous function $L(\alpha, \beta)$ of degree one in two variables $\alpha(x, y)=$ $\sqrt{a_{i j}(x) y^{i} y^{j}}$ and $\beta=b_{i}(x) y^{i}$. The space $R^{n}=(M, \alpha)$ is called the associated Riemannian space with $F^{n}$. We use the following symbols [10]:

$$
\begin{gathered}
r_{i j}=\frac{1}{2}\left(b_{i ; j}+b_{j ; i}\right), s_{i j}=\frac{1}{2}\left(b_{i ; j}-b_{j ; i}\right), \\
s_{j}^{i}=a^{i r} s_{r j}, s_{j}=b_{r} s_{j}^{r} .
\end{gathered}
$$


Further, a Finsler space with an $(\alpha, \beta)$-metric is said to be a Douglas space of the second kind if and only if

$$
B_{m}^{i m}=(n+1) B^{i}-B_{m}^{m} y^{i},
$$

are homogeneous polynomials in $\left(y^{i}\right)$ of degree two, where $B_{m}^{m}$ is given by [7]. Furthermore differentiating the above with respect to $y^{h}, y^{j}$ and $y^{k}$, we get

$$
B_{h j k m}^{i m}=B_{h j k}^{i}=0 .
$$

Thus, we have

Definition 2.2. A Finsler space $F^{n}$ with $(\alpha, \beta)$-metric is said to be a Douglas space of second kind if it satisfies the condition that $B_{m}^{i m}=(n+1) B^{i}-$ $B_{m}^{m} y^{i}$ is homogeneous polynomials in $\left(y^{i}\right)$ of degree two.

Since $L=L(\alpha, \beta)$ is a positively homogeneous function of $\alpha$ and $\beta$ of degree one, we have

$$
\begin{array}{r}
L_{\alpha} \alpha+L_{\beta} \beta=L, L_{\alpha \alpha} \alpha+L_{\alpha \beta} \beta=0, \\
L_{\beta \alpha} \alpha+L_{\beta \beta} \beta=0, L_{\alpha \alpha \alpha} \alpha+L_{\alpha \alpha \beta} \beta=-L_{\alpha \alpha}, \\
L_{\alpha}=\partial L / \partial \alpha, L_{\beta}=\partial L / \partial \beta, L_{\alpha \alpha}=\partial^{2} L / \partial \alpha \partial \alpha, \\
L_{\alpha \beta}=L_{\beta \alpha}=\partial^{2} L / \partial \alpha \partial \beta, L_{\alpha \alpha \alpha}=\partial^{3} L / \partial \alpha \partial \alpha \partial \alpha .
\end{array}
$$

Here we state the following remark for the later frequent use:

Remark 2.3. Throughout the present paper, we say "homogeneous polynomial(s) in $\left(y^{i}\right)$ of degree $r$ " as $h p(r)$ for brevity. Thus $\gamma_{00}^{i}$ is $h p(2)$ and, if the Finsler space with an $(\alpha, \beta)$-metric is a Douglas space of the second kind, then $B_{m}^{i m}$ is $h p(2)$.

\section{Douglas Space of Second Kind with $(\alpha, \beta)$-Metric}

In this section, we deal with the condition for a Finsler space with an $(\alpha, \beta)$ metric to be a Douglas space of the second kind.

Let us consider the function $G^{i}(x, y)$ of $F^{n}$ with an $(\alpha, \beta)$-metric. According to $[9], G^{i}(x, y)$ can be written as

$$
\begin{gathered}
2 G^{i}=\gamma_{00}^{i}+2 B^{i} \\
B^{i}=\left(\alpha \frac{L_{\beta}}{L_{\alpha}}\right) s_{0}^{i}+C^{*}\left[\frac{\beta L_{\beta}}{\alpha L} y^{i}-\frac{\alpha L_{\alpha \alpha}}{L_{\alpha}}\left(\frac{y^{i}}{\alpha}-\frac{\alpha b^{i}}{\beta}\right)\right]
\end{gathered}
$$


where we put

$$
\begin{gathered}
C^{*}=\frac{\alpha \beta\left(r_{00} L_{\alpha}-2 \alpha s_{0} L_{\beta}\right)}{2\left(\beta^{2} L_{\alpha}+\alpha \gamma^{2} L_{\alpha \alpha}\right)}, \\
\gamma^{2}=b^{2} \alpha^{2}-\beta^{2}, b^{i}=a^{i j} b_{j}, b^{2}=a^{i j} b_{i} b_{j} .
\end{gathered}
$$

Since $\gamma_{00}^{i}=\gamma_{j k}^{i}(x) y^{j} y^{k}$ are homogeneous polynomials in $\left(y^{i}\right)$ of degree two, equation (3.1) yields

$$
B^{i j}=\frac{\alpha L_{\beta}}{L_{\alpha}}\left(s_{0}^{i} y^{j}-s_{0}^{j} y^{i}\right)+\frac{\alpha^{2} L_{\alpha \alpha}}{\beta L_{\alpha}} C^{*}\left(b^{i} y^{j}-b^{j} y^{i}\right) .
$$

by means of (2.1) and (3.3), we have the following lemma [11]:

Lemma 3.1. A Finsler space $F^{n}$ with an $(\alpha, \beta)$-metric is a Douglas space if and only if $B^{i j}=B^{i} y^{j}-B^{j} y^{i}$ are $h p(3)$.

Further differentiating (3.3) by $y^{m}$ and contracting $\mathrm{m}$ and $\mathrm{j}$ in the obtained equation, we obtain

$$
\begin{aligned}
B_{m}^{i m} & =\dot{\partial}_{m}\left(\frac{\alpha L_{\beta}}{L_{\alpha}}\right)\left(s_{0}^{i} y^{m}-s_{0}^{m} y^{i}\right)+\frac{\alpha L_{\beta}}{L_{\alpha}} \dot{\partial}_{m}\left(s_{0}^{i} y^{m}-s_{0}^{m} y^{i}\right) \\
& +\dot{\partial}_{m}\left(\frac{\alpha^{2} L_{\alpha \alpha}}{\beta L_{\alpha}}\right) C^{*}\left(b^{i} y^{m}-b^{m} y^{i}\right)+\frac{\alpha^{2} L_{\alpha \alpha}}{\beta L_{\alpha}}\left(\dot{\partial}_{m} C^{*}\right)\left(b^{i} y^{m}-b^{m} y^{i}\right) \\
& +\frac{\alpha^{2} L_{\alpha \alpha}}{\beta L_{\alpha}} C^{*} \dot{\partial}_{m}\left(b^{i} y^{m}-b^{m} y^{i}\right) .
\end{aligned}
$$

Making use of (2.2) and the homogeneity of $\left(y^{i}\right)$, we obtain

$$
\begin{gathered}
\dot{\partial}_{m}\left(\frac{\alpha L_{\beta}}{L_{\alpha}}\right)\left(s_{0}^{i} y^{m}-s_{0}^{m} y^{i}\right)=\left(\frac{\alpha L_{\beta}}{L_{\alpha}}\right) s_{0}^{i}-\frac{\alpha^{2} L L_{\alpha \alpha} s_{0}}{\left(\beta L_{\alpha}\right)^{2}} y^{i}, \\
\frac{\alpha L_{\beta}}{L_{\alpha}} \dot{\partial}_{m}\left(s_{0}^{i} y^{m}-s_{0}^{m} y^{i}\right)=\frac{n \alpha L_{\beta}}{L_{\alpha}} s_{0}^{i}, \\
\dot{\partial}_{m}\left(\frac{\alpha^{2} L_{\alpha \alpha}}{\beta L_{\alpha}}\right) C^{*}\left(b^{i} y^{m}-b^{m} y^{i}\right)=\frac{\gamma^{2}\left\{\alpha L_{\alpha} L_{\alpha \alpha \alpha}+\left(2 L_{\alpha}-\alpha L_{\alpha \alpha}\right) L_{\alpha \alpha}\right\} C^{*}}{\left(\beta L_{\alpha}\right)^{2}} y^{i},
\end{gathered}
$$




$$
\begin{gathered}
\left(\dot{\partial}_{m} C^{*}\right) y^{m}=2 C^{*}, \\
\left(\dot{\partial}_{m} C^{*}\right) b^{m}=\frac{1}{2 \alpha \beta \Omega^{2}}\left[\Omega \left\{\beta\left(\gamma^{2}+2 \beta^{2}\right) M+2 \alpha^{2} \beta^{2} L_{\alpha} r_{0}\right.\right. \\
\left.-\alpha \beta \gamma^{2} L_{\alpha \alpha} r_{00}-2 \alpha\left(\beta^{3} L_{\beta}+\alpha^{2} \gamma^{2} L_{\alpha \alpha}\right) s_{0}\right\} \\
\left.-\alpha^{2} \beta M\left(2 b^{2} \beta^{2} L_{\alpha}-\gamma^{4} L_{\alpha \alpha \alpha}-b^{2} \alpha \gamma^{2} L_{\alpha \alpha}\right)\right], \\
\frac{\alpha^{2} L_{\alpha \alpha}}{\beta L_{\alpha}} C^{*} \dot{\partial}_{m}\left(b^{i} y^{m}-b^{m} y^{i}\right)=\frac{(n-1) \alpha^{2} L_{\alpha \alpha} C^{*}}{\beta L_{\alpha}} b^{i},
\end{gathered}
$$

where

$$
M=\left(r_{00} L_{\alpha}-2 \alpha s_{0} L_{\beta}\right), Y_{i}=a_{i r} y^{r}, s_{00}=0, b^{r} s_{r}=0, a^{i j} s_{i j}=0 .
$$

Substituting (3.5), (3.6), (3.7), (3.8), (3.9) and (3.10) into (3.4), we have

$$
\begin{gathered}
B_{m}^{i m}=\frac{(n+1) \alpha L_{\beta}}{L_{\alpha}} s_{0}^{i}+\frac{\alpha\left\{(n+1) \alpha^{2} \Omega L_{\alpha \alpha} b^{i}+\beta \gamma^{2} A y^{i}\right\}}{2 \Omega^{2}} r_{00}- \\
\frac{\alpha^{2}\left\{(n+1) \alpha^{2} \Omega L_{\beta} L_{\alpha \alpha} b^{i}+B y^{i}\right\}}{L_{\alpha} \Omega^{2}} s_{0}-\frac{\alpha^{3} L_{\alpha \alpha} y^{i}}{\Omega} r_{0}
\end{gathered}
$$

where

$$
\begin{aligned}
& \Omega=\left(\beta^{2} L_{\alpha}+\alpha \gamma^{2} L_{\alpha \alpha}\right), \Omega \neq 0 \\
& A=\alpha L_{\alpha} L_{\alpha \alpha \alpha}+3 L_{\alpha} L_{\alpha \alpha}-3 \alpha\left(L_{\alpha \alpha}\right)^{2}, \\
& B=\alpha \beta \gamma^{2} L_{\alpha} L_{\beta} L_{\alpha \alpha \alpha}+\beta\left\{\left(3 \gamma^{2}-\beta^{2}\right) L_{\alpha}-4 \alpha \gamma^{2} L_{\alpha \alpha}\right\} L_{\beta} L_{\alpha \alpha}+\Omega L L_{\alpha \alpha} .
\end{aligned}
$$

We use the following result [7];

Theorem 3.2. The necessary and sufficient condition for a Finsler space $F^{n}$ with an $(\alpha, \beta)$-metric to be a Douglas space of the second kind is that, $B_{m}^{i m}$ are homogeneous polynomials in $\left(y^{m}\right)$ of degree two, where $B_{m}^{i m}$ is given by (3.12) and (3.13), provided that $\Omega \neq 0$.

\section{Conformal Change of Douglas Space of Second Kind with $(\alpha, \beta)$-Metric}

In the present section, we derive the condition on conformal change, so that a Douglas space of second kind is conformally transformed to a Douglas space of second kind. 
Let $F^{n}=(M, L)$ and $\bar{F}^{n}=(M, \bar{L})$ be two Finsler space on the same underlying manifold $M$. If we have a function $\sigma(x)$ in each coordinate neighbourhood of $M$ such that $\bar{L}(x, y)=e^{\sigma} L(x, y)$, then $F^{n}$ is called conformal to $\bar{F}^{n}$ and the change $L \rightarrow \bar{L}$ of metric is called conformal change.

As to $(\alpha, \beta)$-metric, $\bar{L}=e^{\sigma} L(\alpha, \beta)$, is equivalent to $\bar{L}=L\left(e^{\sigma} \alpha, e^{\sigma} \beta\right)$ by homogeneity. Therefore, a conformal change of $(\alpha, \beta)$-metric is expressed as $(\alpha, \beta) \rightarrow(\bar{\alpha}, \bar{\beta})$, where $\bar{\alpha}=e^{\sigma} \alpha, \bar{\beta}=e^{\sigma} \beta$. Therefore, we have

$$
\begin{gathered}
\bar{a}_{i j}=e^{2 \sigma} a_{i j}, \bar{b}_{i}=e^{\sigma} b_{i} \\
\bar{a}^{i j}=e^{-2 \sigma} a^{i j}, \bar{b}^{i}=e^{-\sigma} b^{i}
\end{gathered}
$$

and $b^{2}=a^{i j} b_{i} b_{j}=\bar{a}^{i j} \bar{b}_{i} \bar{b}_{j}$. Thus we state the following:

Proposition 4.1. A Finsler space with $(\alpha, \beta)$-metric and the length $b$ of $b_{i}$ with respect to the Riemannian metric $\alpha$ is invariant under any conformal change of $(\alpha, \beta)$-metric.

From (4.1), it follows that the conformal change of Cristoffel symbols is given by [4];

$$
\bar{\gamma}_{j k}^{i}=\gamma_{j k}^{i}+\delta_{j}^{i} \sigma_{k}+\delta_{k}^{i} \sigma_{j}-\sigma^{i} a_{j k},
$$

where $\sigma_{j}=\partial_{j} \sigma$ and $\sigma^{i}=a^{i j} \sigma_{j}$.

From (4.1) and (4.2), we have the following identities:

$$
\begin{aligned}
\bar{\nabla}_{j} \bar{b}_{i} & =e^{\sigma}\left(\nabla_{j} b_{i}+\rho a_{i j}-\sigma_{i} b_{j}\right), \\
\bar{r}_{i j} & =e^{\sigma}\left[r_{i j}+\rho a_{i j}-\frac{1}{2}\left(b_{i} \sigma_{j}+b_{j} \sigma_{i}\right)\right], \bar{s}_{i j}=e^{\sigma}\left[s_{i j}+\frac{1}{2}\left(b_{i} \sigma_{j}-b_{j} \sigma_{i}\right)\right], \\
\bar{s}_{j}^{i} & =e^{-\sigma}\left[s_{j}^{i}+\frac{1}{2}\left(b^{i} \sigma_{j}-b_{j} \sigma^{i}\right)\right], \bar{s}_{j}=s_{j}+\frac{1}{2}\left(b^{2} \sigma_{j}-\rho b_{j}\right),
\end{aligned}
$$

where $\rho=\sigma_{r} b^{r}$.

From (4.2) and (4.3), we can easily obtain the following:

$$
\begin{aligned}
\bar{\gamma}_{00}^{i} & =\gamma_{00}^{i}+2 \sigma_{0} y^{i}-\alpha^{2} \sigma_{j}, \bar{r}_{00}=e^{\sigma}\left(r_{00}+\rho \alpha^{2}-\sigma_{0} \beta\right), \\
\bar{s}_{0}^{i} & =e^{-\sigma}\left[s_{0}^{i}+\frac{1}{2}\left(\sigma s_{0} b^{i}-\beta \sigma^{i}\right)\right], \quad \bar{s}_{0}=s_{0}+\frac{1}{2}\left(\sigma_{0} b^{i}-\rho \beta\right) .
\end{aligned}
$$

Next, we find the conformal change of $B^{i j}$ given in (3.3), since $\bar{L}(\alpha, \beta)=$ $e^{\sigma} L(\alpha, \beta)$, and

$$
\bar{L}_{\bar{\alpha}}=L_{\alpha}, \bar{L}_{\bar{\alpha} \bar{\alpha}}=e^{-\sigma} L_{\alpha \alpha}, \bar{L}_{\bar{\beta}}=L_{\beta}, \bar{\gamma}^{2}=e^{2 \sigma} \gamma^{2} .
$$


By using (3.2), (4.4), (4.5) and lemma (3.1), we obtain

$$
\bar{C}^{*}=e^{\sigma}\left(C^{*}+D^{*}\right),
$$

where

$$
D^{*}=\frac{\alpha \beta\left\{\left(\beta \alpha^{2}-\sigma_{0} \beta\right) L_{\alpha}-\alpha\left(b^{2} \sigma_{0}-\rho \beta\right) L_{\beta}\right\}}{2\left(\beta^{2} L_{\alpha}+\alpha \gamma^{2} L_{\alpha \alpha}\right)} .
$$

Here under the conformal change $B^{i j}$ can be written as:

$$
\begin{aligned}
\bar{B}^{i j} & =\frac{\alpha L_{\beta}}{L_{\alpha}}\left(s_{0}^{i} y^{j}-s_{0}^{i} y^{i}\right)+\frac{\alpha^{2} L_{\alpha \alpha}}{\beta L_{\alpha}} C^{*}\left(b^{i} y^{j}-b^{j} y^{i}\right) \\
& +\left(\frac{\alpha \sigma_{0} L_{\beta}}{L_{\alpha}}+\frac{\alpha^{2} L_{\alpha \alpha}}{\beta L_{\alpha}} D^{*}\right)\left(b^{i} y^{j}-b^{j} y^{i}\right)-\frac{\alpha \beta L_{\beta}}{2 L_{\alpha}}\left(\sigma^{i} y^{j}-\sigma^{j} y^{i}\right), \\
& =B^{i j}+C^{i j},
\end{aligned}
$$

where

$$
C^{i j}=\left(\frac{\alpha \sigma_{0} L_{\beta}}{L_{\alpha}}+\frac{\alpha^{2} L_{\alpha \alpha}}{\beta L_{\alpha}} D^{*}\right)\left(b^{i} y^{j}-b^{j} y^{i}\right)-\frac{\alpha \beta L_{\beta}}{2 L_{\alpha}}\left(\sigma^{i} y^{j}-\sigma^{j} y^{i}\right) .
$$

From the equation (3.13), it is clear that

$$
\bar{\Omega}=e^{2 \sigma} \Omega, \bar{A}=e^{-\sigma} A, \bar{B}=e^{2 \sigma} B .
$$

Now we apply conformal transformation to $B_{m}^{i m}$, and obtain

$$
\bar{B}_{m}^{i m}=B_{m}^{i m}+K_{m}^{i m},
$$

where

$$
\begin{aligned}
2 K_{m}^{i m} & =\frac{(n+1) \alpha L_{\beta}}{L_{\alpha}}\left(\sigma_{0} b^{i}-\beta \sigma^{i}\right)+\frac{(n+1) \alpha^{3} \Omega L_{\alpha \alpha} b^{i}+\alpha \beta \gamma^{2} A y^{i}}{\Omega^{2}}\left(\rho \alpha^{2}-\sigma_{0} \beta\right) \\
& -\left[\frac{\alpha^{2}(n+1) \alpha^{2} \Omega L_{\beta} L_{\alpha \alpha} b^{i}+B y^{i}}{L_{\alpha} \Omega^{2}}-\frac{\alpha^{3} L_{\alpha \alpha} y^{i}}{\Omega}\right]\left(b^{2} \sigma_{0}-\rho \beta\right) .
\end{aligned}
$$

Hence, we have the following:

Theorem 4.2. The necessary and sufficient condition for a Douglas space of second kind with $(\alpha, \beta)$-metric to be a Douglas space of second kind under conformal transformation, is that $K_{m}^{i m}(x)$ are homogenous polynomial in $\left(y^{m}\right)$ of degree two. 


\section{Conformal Change of Douglas Space of Second Kind with Certain $(\alpha, \beta)$-Metric}

The important examples of Finsler space with $(\alpha, \beta)$-metric are Randers space, Kropina space, generalized Kropina space and Matsumoto space. The conditions for Douglas space of second kind with Randers metric and Kropina metric to be a Douglas space of second kind under conformal change have been obtained in [13].

In this section, we extend the study on conformal change of Douglas space of second kind and obtain the conditions for Douglas space of second kind with generalized Kropina metric $L=\frac{\alpha^{m+1}}{\beta^{m}},(m \neq 0,-1)$ and Matsumoto metric $L=\frac{\alpha^{2}}{\alpha-\beta}$ to be a Douglas space of second kind under conformal change.

\subsection{Conformal Change of Douglas Space of Second Kind with Generalized Kropina Metric}

Let us consider the Douglas space of second kind with $(\alpha, \beta)$-metric $L(\alpha, \beta)=$ $\frac{\alpha^{m+1}}{\beta^{m}}(m \neq 0,-1)$, known as generalized Kropina metric. Then we have

$$
\begin{array}{r}
L_{\alpha}=(m+1) \frac{\alpha^{m}}{\beta^{m}}, \quad L_{\beta}=-m \frac{\alpha^{m+1}}{\beta^{m+1}}, \\
L_{\alpha \alpha}=m(m+1) \frac{\alpha^{m-1}}{\beta^{m}}, \quad L_{\alpha \alpha \alpha}=m\left(m^{2}-1\right) \frac{\alpha^{m-2}}{\beta^{m}} .
\end{array}
$$

Hence, from (3.13) we have

$$
\begin{aligned}
& \Omega=(m+1)\left\{(1-m) \beta^{2}+b^{2} m \alpha^{2}\right\} \frac{\alpha^{m}}{\beta^{m}}, \\
& A=2 m(m+1)^{2}(1-m) \frac{\alpha^{2 m-1}}{\beta^{2 m}}, \\
& B=m(m+1)^{2}\left\{(3 m-1) b^{2} m \alpha^{2}-(3 m+1)(m-1) \beta^{2}\right\} \frac{\alpha^{3 m}}{\beta^{3 m}} .
\end{aligned}
$$

Thus, from (4.7), $K_{m}^{i m}$ is reduces to

$$
\begin{aligned}
2 K_{m}^{i m} & =-(n+1) \frac{m \alpha^{2}}{(m+1) \beta}\left(\sigma_{0} b^{i}-\beta \sigma^{i}\right)+\frac{(n+1) m \alpha^{2} b^{i}}{\left\{(1-m) \beta^{2}+b^{2} m \alpha^{2}\right\}}\left(\rho \alpha^{2}-\sigma_{0} \beta\right) \\
& +\frac{2\left(b^{2} \alpha^{2}-\beta^{2}\right) m(1-m) \beta y^{i}}{\left\{(1-m) \beta^{2}+b^{2} m \alpha^{2}\right\}^{2}}\left(\rho \alpha^{2}-\sigma_{0} \beta\right)
\end{aligned}
$$




$$
\begin{aligned}
& \frac{-m^{2}(n+1) \alpha^{4} b^{i}}{(m+1)\left\{(1-m) \beta^{2}+b^{2} m \alpha^{2}\right\} \beta}\left(b^{2} \sigma_{0}-\rho \beta\right) \\
& -\frac{m\left\{(3 m-1) b^{2} m \alpha^{2}-(3 m+1)(m-1) \beta^{2}\right\} \alpha^{2} y^{i}}{(m+1)\left\{(1-m) \beta^{2}+b^{2} m \alpha^{2}\right\}^{2}}\left(b^{2} \sigma_{0}-\rho \beta\right) \\
& +\frac{\left.m \alpha^{2} y^{i}\right)}{\left\{(1-m) \beta^{2}+b^{2} m \alpha^{2}\right\}}\left(b^{2} \sigma_{0}-\rho \beta\right) .
\end{aligned}
$$

which shows that $K_{m}^{i m}$ is $\operatorname{hp}(2)$. Hence

Theorem 5.1. The Douglas space of second kind with generalized Kropina metric is conformally transformed to a Douglas space of second kind.

\subsection{Conformal Change of Douglas Space of Second Kind with Matsumoto Metric}

Let us consider the Douglas space of second kind with $(\alpha, \beta)$-metric $L(\alpha, \beta)=$ $\frac{\alpha^{2}}{\alpha-\beta}$, known as Matsumoto metric. Then we have

$$
\begin{gathered}
L_{\alpha}=\frac{\alpha^{2}-2 \alpha \beta}{(\alpha-\beta)^{2}}, \quad L_{\beta}=\frac{\alpha^{2}}{(\alpha-\beta)^{2}}, \\
L_{\alpha \alpha}=\frac{2 \beta^{2}}{(\alpha-\beta)^{3}}, \quad L_{\alpha \alpha \alpha}=\frac{-6 \beta^{2}}{(\alpha-\beta)^{4}} .
\end{gathered}
$$

Hence, from (3.13) we have

$$
\begin{aligned}
& A=\frac{-6 \alpha^{2} \beta^{3}}{(\alpha-\beta)^{6}}, \quad \Omega=\frac{\alpha^{2} \beta^{2}\left[\left(1+2 b^{2}\right) \alpha-3 \beta\right]}{(\alpha-\beta)^{3}}, \\
& B=\frac{2 \alpha^{2} \beta^{2}\left[\left(1-b^{2}\right) \alpha^{2}-\left(4 b^{2}+5\right) \alpha \beta+9 \beta^{2}\right]}{(\alpha-\beta)^{8}} .
\end{aligned}
$$

Thus, from (4.7), $K_{m}^{i m}$ is reduces to

$$
\begin{aligned}
2 K_{m}^{i m} & =\frac{(n+1) \alpha^{2}}{(\alpha-2 \beta)}\left(\sigma_{0} b^{i}-\beta \sigma^{i}\right)+\frac{2(n+1) \alpha b^{i}}{\left\{\left(1+2 b^{2}\right) \alpha-3 \beta\right\}}\left(\rho \alpha^{2}-\sigma_{0} \beta\right) \\
& -\frac{6\left(b^{2} \alpha^{2}-\beta^{2}\right) y^{i}}{\alpha\left\{\left(1+2 b^{2}\right) \alpha-3 \beta\right\}^{2}}\left(\rho \alpha^{2}-\sigma_{0} \beta\right)- \\
& \frac{2(n+1)\left\{\left(1+2 b^{2}\right) \alpha-3 \beta\right\} \alpha^{3} b^{i}}{(\alpha-2 \beta)\left\{\left(1+2 b^{2}\right) \alpha-3 \beta\right\}^{2}}\left(b^{2} \sigma_{0}-\rho \beta\right) \\
& -\frac{2 \alpha y^{i}\left\{\left(1-b^{2}\right) \alpha^{2}+9 \beta^{2}-\left(4 b^{2}+5\right) \alpha \beta\right\}}{(\alpha-2 \beta)\left\{\left(1+2 b^{2}\right) \alpha-3 \beta\right\}^{2}}\left(b^{2} \sigma_{0}-\rho \beta\right)
\end{aligned}
$$




$$
+\frac{2 \alpha y^{i}}{\left\{\left(1+2 b^{2}\right) \alpha-3 \beta\right\}}\left(b^{2} \sigma_{0}-\rho \beta\right) .
$$

which shows that $K_{m}^{i m}$ is $\mathrm{hp}(2)$. Hence

Theorem 5.2. The Douglas space of second kind with Matsumoto metric is conformally transformed to a Douglas space of second kind.

\section{References}

[1] Antonelli, P. L., Ingarden, R. S. and Matsumoto, M., The Theory of sprays and Finsler spaces with Applications in Physics and Biology, Kluwer Acad. Publ., Dordrecht, (1993).

[2] Bácsó, S. and Matsumoto, M., On Finsler spaces of Douglas type. A generalization of the notion of Berwald space, Publ. Math. Debrecen, 51, (1997), 385-406.

[3] Berwald, L., On Cartan and Finsler geometries, III, Two-dimensional Finsler spaces with rectilinear extremal, Ann. of Math., 42, (1941), 84112 .

[4] M. Hashiguchi, On Conformal transformations of diffrence tensors of Finsler space, J. Math. Kyoto Univ., 16, (1976), 25-50.

[5] M. S. Knebelman, Conformal geometry of generalized metric spaces, Proc. Nat. Acad. Sci. USA, 15, (1929), 376-379.

[6] I. Y. Lee, On weakly-Berwald spaces of $(\alpha, \beta)$-metric, Bull. Korean Math. Soc., 43, 2, (2006), 425-441.

[7] I. Y. Lee, Douglas space of the second kind of Finsler space with a Matsumoto metric, Journal of the Chungcheong Mathematical society, 21, 2, (2008), 209-221.

[8] Y. D. Lee, Conformal transformations of diffrence tensors of Finsler space with an ( $\alpha, \beta)$-metric, Comm. Korean Math. Soc., 21, 4, (1997), 975-984.

[9] M. Matsumoto, The Berwald connection of a Finsler space with $(\alpha, \beta)$ metric, Tensor, N. S., 50 (1991), 18-21.

[10] M. Matsumoto, Theory of Finsler spaces with $(\alpha, \beta)$-metric, Rep. On Math. Phys., 31, (1992), 43-83. 
[11] M. Matsumoto, Finsler spaces with $(\alpha, \beta)$-metric of Douglas type, Tensor, N. S., 60, (1998), 123-134.

[12] S. K. Narasimhamurthy, D. M. Vasantha and Ajith, Conformal change of Douglas space with special $(\alpha, \beta)$-metric, Investigations in Mathematical Sciences, 2, 1, (2012), 290-301.

[13] S. K. Narasimhamurthy, Ajith and C. S. Bagewadi, Conformal change of Douglas space of second kind with $(\alpha, \beta)$-metric, Journal of Math. Analysis, 3, 2, (2012), 25-30.

[14] B. N. Prasad, B. N. Gupta and D. D. Singh, On Conformal transformation in Finsler spaces with an $(\alpha, \beta)$-metric, Indian J. pure appl. Math., 18, 4, (1987), 290-301. 